\title{
Ten-Year Follow-Up of Transcranial Magnetic Stimulation Study in a Patient With Congenital Mirror Movements: A Case Report
}

\author{
Eu-Deum Kim, $\mathrm{MD}^{1}$, Gi-Wook Kim, MD, $\mathrm{PhD}^{1,2}$, Yu Hui Won, $\mathrm{MD}, \mathrm{PhD}^{1,2}$, \\ Myoung-Hwan Ko, MD, PhD ${ }^{1,2}$, Jeong-Hwan Seo, MD, PhD ${ }^{1,2}$, Sung-Hee Park, MD, PhD ${ }^{1,2}$ \\ ${ }^{1}$ Department of Physical Medicine and Rehabilitation, Chonbuk National University Medical School, Jeonju;
} ${ }^{2}$ Research Institute of Clinical Medicine of Chonbuk National University-Biomedical Research Institute of Chonbuk National University Hospital, Jeonju, Korea

Most studies concerning congenital mirror movements (CMMs) have been focused on the motor organization in the distal hand muscles exclusively. To the best of our knowledge, there is no data on motor organization pattern of lower extremities, and a scarcity of data on the significance of forearm and arm muscles in CMMs. Here, we describe the case of a 19-year-old boy presenting mirror movements. In these terms, a 10-year transcranial magnetic stimulation study demonstrated that the motor organization pattern of the arm muscles was different from that of distal hand and forearm muscles even in the same upper extremity, and that the lower extremities showed the same pathways as healthy children. Moreover, in this case, an ipsilateral motor evoked potentials (MEPs) for distal hand muscles increased in amplitude with age, even though the intensity of mirror movements decreased. In the arm muscles, however, it was concluded that the contralateral MEPs increased in amplitude with age.

Keywords Pyramidal tracts, Evoked potentials, Motor, Synkinesis

\section{INTRODUCTION}

To understand the motor control of the hand in patients with congenital mirror movements (CMMs), investigation of motor organization in distal hand muscles is important to develop viable interventions to improve pa- tient outcomes. However, forearm and arm muscles also contribute to hand motor function and must be considered in these cases. Most studies concerning CMMs have been focused on the motor organization in the distal hand muscles only, and confirmed the dominance of the ipsilateral corticospinal pathways in those muscles. To

Received July 3, 2018; Accepted September 6, 2018

Corresponding author: Sung-Hee Park

Department of Physical Medicine and Rehabilitation, Chonbuk National University Medical School, 20 Geonji-ro, Deokjin-gu, Jeonju 54907, Korea. Tel: +82-63-250-2299, Fax: +82-63-254-4145, E-mail: shpark0130@jbnu.ac.kr

ORCID: Eu-Deum Kim (http://orcid.org/0000-0001-6659-7207); Gi-Wook Kim (http://orcid.org/0000-0002-1628-8382); Yu Hui Won (http://orcid. org/0000-0003-2007-9652); Myoung-Hwan Ko (http://orcid.org/0000-0002-0566-3677); Jeong-Hwan Seo (http://orcid.org/0000-0002-6915-6674); Sung-Hee Park (http://orcid.org/0000-0002-4743-2551).

(c) This is an open-access article distributed under the terms of the Creative Commons Attribution Non-Commercial License (http://creativecommons.org/ licenses/by-nc/4.0) which permits unrestricted noncommercial use, distribution, and reproduction in any medium, provided the original work is properly cited. Copyright $\odot 2019$ by Korean Academy of Rehabilitation Medicine 
the best of our knowledge, there is scarce data on forearm and arm muscles, and no data on the lower extremities in these cases [1].

In a previous study, we probed the ipsilateral corticospinal pathway in a patient with CMMs by examining neurophysiologic findings for the distal hand muscles [2]. In this study, we aimed to investigate the motor organization of the forearm and arm muscles as well as the distal hand muscles and lower extremities in the same patient, as evaluated via a transcranial magnetic stimulation (TMS) study repeated over a period of 10 years. We hoped by utilizing this approach that we would be in the position to gain an insight into the pathophysiology of CMMs and also the impact of development on the disorder.

\section{CASE REPORT}

The patient first visited our hospital at the age of 9, presenting with mirror movements [2]. It was revealed that involuntary symmetrical movements occurred in the opposite hand when he moved his hand. Therefore, he had difficulty in tying his shoelace and fastening a button such as on a shirt. He received occupational therapy and went to a general school, but his educational achievement was very low. At the age of 19 , his mirror movements were more reduced than had been noted before. He was able to perform dissociated movements with his hands such as typing and assembling bolts and nuts. However, he still displayed a marked level of clumsiness and low grip strength in both hands. In the 9-hole pegboard test, it was noted that he took 26 seconds for each hand (normal: 16.41 \pm 1.65 seconds for the right hand, $17.53 \pm 1.73$ seconds for the left hand) and his grip strength for each hand was $26 \mathrm{~kg}$ (normal: $43.1-49.0 \mathrm{~kg}$ for each hand).

His mirror movements were recently reassessed ac- cording to the Woods and Teuber scale [3]. In this case, the mirror movements were persistently observed in the upper extremities, particularly the distal hands, but not in the lower extremities. The intensity of mirror movements was decreased at age of 19 , but their frequency was not reduced. The scores for lifting a finger, opening and clenching the fist, sequential finger-thumb opposition, flexing the wrist, and abducting the arm were all noted at an evaluated number 1 , where they were 2 or 3 at the age of 9 .

Equally important, it is noted that the brain magnetic resonance imaging showed no abnormal findings. Similarly, the diffusion tensor images (DTI) were acquired using a Verio 3.0T system (Siemens, Erlangen, Germany) equipped with a 12-channel sensitivity encoding (SENSE) head coil for single-shot echo planar imaging. The imaging parameters utilized were: echo time $=93 \mathrm{~ms}$, repetition time $=7,900 \mathrm{~ms}$, field of view $=230 \mathrm{~mm} \times 230 \mathrm{~mm}$, sampling matrix size $=128 \times 128$ reconstructed with homodyne processing to $256 \times 256$, SENSE factor $=3$, EPI $=128$, and $b$ value $=1,000 \mathrm{~s} / \mathrm{mm}^{2}$. As a result, we acquired 47 contiguous, $3.0 \mathrm{~mm}$ thick slices parallel to the anterior commissure-posterior commissure line in 30 different diffusion directions. We therefore performed a tractography on the basis of fiber assignment by continuous tracking (FACT). The thresholds of the tracking termination were noted at 0.2 for the fractional anisotropy (FA) and $60^{\circ}$ for the angle. Furthermore, for fiber tracking of the corticospinal tract (CST), two region of interest (ROI) were drawn on color-coded two-demensional FA map. For this purpose, a seed ROI was drawn at the CST portion in the anterior mid-pons and the target ROI in the anterior lower pons. As has been noted, the diffusion tensor tractography demonstrated normal symmetrical crossed CST (Fig. 1).

In this study, we performed a TMS study spanning 10 years from aged 9 to 19 . The possible physical and psy-
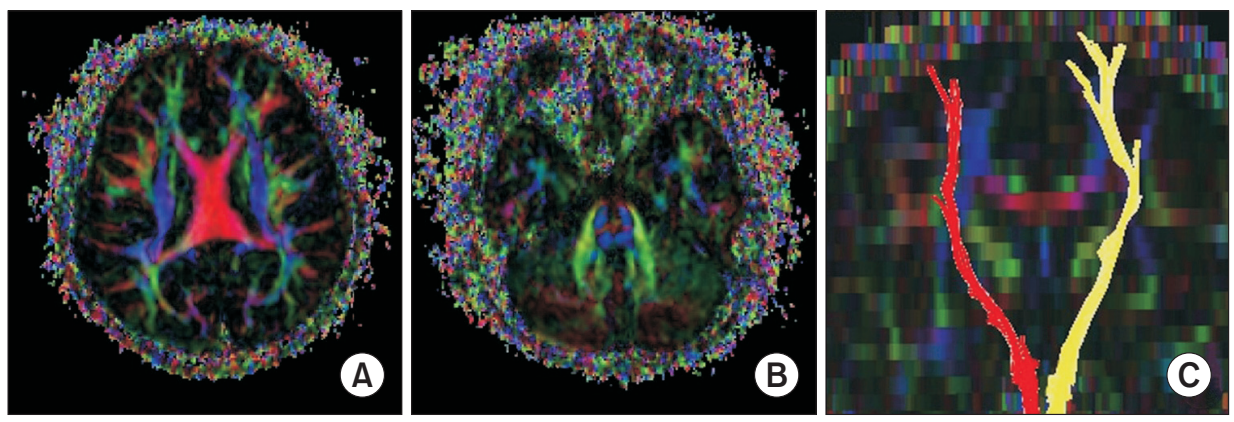

Fig. 1. Axial color-coded fractional anisotropy map demonstrating well-defined blue color corticospinal tract (CST) at the level of the cerebral cortex (A) and pons (B). The normal connectivity of whole CST was confirmed by diffusion tensor tractography (C). 
chological complications from the study were explained to the patient, who gave written informed consent to participate in the study. The TMS system utilized was a MagPro (MagVenture, Lucernemarken, Denmark), and figure-8 type magnetic coils (70 $\mathrm{mm}$ in diameter) were used to stimulate the primary motor cortex. The recordings of ipsilateral motor evoked potentials (iMEPs) and contralateral motor evoked potentials (cMEPs) were made simultaneously at the bilateral first dorsal interosseous (FDI), extensor carpi radialis (ECR), biceps brachii (BB), deltoid, tibialis anterior, gastrocnemius and vastus medialis. Additionally, the FDI, ECR, and BB plus deltoid represent the distal hand, forearm, and arm muscles, respectively. In this case, the stimulation intensity was set at $110 \%$ of the resting motor threshold. It is noted that each hemisphere was stimulated four times, and the shortest latency and average peak to peak amplitudes were used for analysis.

In this case, iMEPs were evoked from all upper extremity muscles. At the FDI and ECR the amplitude and latency of iMEPs were noted as being higher and shorter than those of cMEPs (Table 1). Therefore, the ratio of the amplitude of the iMEPs to the cMEPs (iMEP/cMEP) was greater than 1 at these points (Table 2). Additionally, the reverse case was observed in the $\mathrm{BB}$ and deltoid muscles. At the $\mathrm{BB}$ and deltoid the amplitude and the latency of iMEPs were lower and longer than those of cMEPs (Table 1). It is concluded that there were no ipsilateral motor evoked responses in the lower extremities (Table 3).

The iMEPs in distal hand muscles increased in amplitude as the patient grew older, despite the decrement in intensity of the evaluated mirror movements. In the arm muscles, however, the cMEPs were seen to have increased in amplitude. Likewise, for forearm muscles, iMEPs were persistently dominant even though the $\mathrm{iMEP} / \mathrm{cMEP}$ ratio was lower than for the distal hand muscles (Fig. 2).

In this study, we performed whole exome sequencing to look for gene mutations that have been associated with CMMs, specifically DCC, RAD51, PROK2, SEMA3A, SOX10, FGF8, CEP152, CHD7, FGFR1, TACR3, KISS1R, NSMF, PROKR2, WDR11, CREBBP, EP300, HS6ST1, HESX1, and GDF6 [4]. However, it is noted that no pathogenic variant relating to CMMs was found, and there was no family history of mirror movements, which reflects that this case is considered as a sporadic situation.

Table 1. Latencies of ipsilateral and contralateral MEPs in the upper extremities

\begin{tabular}{|c|c|c|c|c|c|}
\hline & \multirow{2}{*}{ Age (yr) } & \multicolumn{2}{|c|}{ Left hemisphere } & \multicolumn{2}{|c|}{ Right hemisphere } \\
\hline & & iMEP (ms) & cMEP (ms) & iMEP (ms) & cMEP (ms) \\
\hline \multirow[t]{4}{*}{ FDI } & 13 & $19.2 \pm 0.0$ & $19.2 \pm 0.0$ & $19.4 \pm 0.8$ & $21.1 \pm 0.8$ \\
\hline & 14 & $20.3 \pm 0.3$ & $20.3 \pm 0.3$ & $21.2 \pm 0.4$ & $32.2 \pm 0.9$ \\
\hline & 15 & $20.1 \pm 0.1$ & $20.4 \pm 0.3$ & $22.1 \pm 0.3$ & $28.8 \pm 0.1$ \\
\hline & 19 & $20.1 \pm 0.3$ & $20.9 \pm 0.1$ & $22.0 \pm 0.6$ & $30.2 \pm 0.6$ \\
\hline \multirow[t]{4}{*}{ ECR } & 13 & $15.2 \pm 0.2$ & $15.2 \pm 0.2$ & $15.2 \pm 0.5$ & $15.4 \pm 0.3$ \\
\hline & 14 & $17.0 \pm 0.0$ & $18.5 \pm 0.0$ & $14.4 \pm 0.1$ & $14.5 \pm 0.0$ \\
\hline & 15 & $16.2 \pm 0.2$ & $19.2 \pm 0.6$ & $16.0 \pm 0.1$ & $21.2 \pm 0.7$ \\
\hline & 19 & $15.2 \pm 0.1$ & $15.3 \pm 0.2$ & $16.5 \pm 0.1$ & $16.8 \pm 0.1$ \\
\hline \multirow[t]{4}{*}{ BB } & 13 & $13.1 \pm 0.4$ & $14.0 \pm 0.5$ & $14.5 \pm 0.4$ & $13.1 \pm 0.1$ \\
\hline & 14 & $16.4 \pm 0.2$ & $15.0 \pm 0.0$ & $16.0 \pm 0.4$ & $12.7 \pm 0.2$ \\
\hline & 15 & $17.0 \pm 0.6$ & $13.5 \pm 0.3$ & $15.9 \pm 0.7$ & $12.9 \pm 0.7$ \\
\hline & 19 & $15.6 \pm 0.1$ & $12.8 \pm 0.6$ & $16.1 \pm 0.2$ & $14.1 \pm 0.2$ \\
\hline \multirow[t]{4}{*}{ Del } & 13 & $12.3 \pm 0.2$ & $12.2 \pm 0.1$ & $13.8 \pm 0.5$ & $12.3 \pm 0.4$ \\
\hline & 14 & $12.5 \pm 0.0$ & $12.5 \pm 0.0$ & $13.0 \pm 0.0$ & $12.1 \pm 0.1$ \\
\hline & 15 & $15.3 \pm 0.6$ & $14.4 \pm 0.8$ & $15.0 \pm 0.7$ & $12.6 \pm 0.4$ \\
\hline & 19 & $15.7 \pm 0.4$ & $11.1 \pm 0.2$ & $12.7 \pm 0.3$ & $11.8 \pm 0.3$ \\
\hline
\end{tabular}

Values are presented as mean \pm standard deviation.

iMEP, ipsilateral motor evoked potential; cMEP, contralateral motor evoked potential; FDI, first dorsal interosseous; ECR, extensor carpi radialis; BB, biceps brachii; Del, deltoid. 
Table 2. iMEPs/cMEPs ratios and the frequency of ipsilateral MEPs of upper extremities

\begin{tabular}{|c|c|c|c|c|c|}
\hline & \multirow{2}{*}{ Age (yr) } & \multicolumn{2}{|c|}{ Left hemisphere } & \multicolumn{2}{|c|}{ Right hemisphere } \\
\hline & & iMEPs/cMEPs ratio & Freq. of iMEPs (\%) & iMEPs/cMEPs ratio & Freq. of iMEPs (\%) \\
\hline \multirow[t]{4}{*}{ FDI } & 13 & 5.0 & 100 & 6.3 & 100 \\
\hline & 14 & 4.4 & 100 & 9.0 & 100 \\
\hline & 15 & 4.7 & 100 & 6.0 & 100 \\
\hline & 19 & 3.7 & 100 & 10.8 & 100 \\
\hline \multirow[t]{4}{*}{ ECR } & 13 & 1.4 & 100 & 2.7 & 100 \\
\hline & 14 & 1.2 & 100 & 1.9 & 100 \\
\hline & 15 & 2.0 & 100 & 2.2 & 100 \\
\hline & 19 & 1.4 & 100 & 2.2 & 100 \\
\hline \multirow[t]{4}{*}{ BB } & 13 & 1.2 & 100 & 0.7 & 100 \\
\hline & 14 & 0.5 & 100 & 0.5 & 100 \\
\hline & 15 & 0.9 & 100 & 0.2 & 100 \\
\hline & 19 & 0.1 & 100 & 0.3 & 100 \\
\hline \multirow[t]{4}{*}{ Del } & 13 & 0.6 & 100 & 0.5 & 100 \\
\hline & 14 & 0.8 & 100 & 0.3 & 100 \\
\hline & 15 & 0.6 & 100 & 0.4 & 100 \\
\hline & 19 & 0.6 & 100 & 0.4 & 100 \\
\hline
\end{tabular}

MEPs, motor evoked potentials; iMEPs/cMEPs ratio, the ratio of amplitude of ipsilateral MEPs to contralateral MEPs; Freq. of iMEPs, the percentage of trials in which iMEPs are elicited by stimulation of the unilateral hemisphere; FDI, first dorsal interosseous; ECR, extensor carpi radialis; BB, biceps brachii; Del, deltoid.

Table 3. Latency and amplitude of contralateral MEPs for the lower extremities

\begin{tabular}{|c|c|c|c|c|c|c|c|}
\hline & \multirow{3}{*}{$\begin{array}{l}\text { Age } \\
(y r)\end{array}$} & \multicolumn{3}{|c|}{ Left hemisphere } & \multicolumn{3}{|c|}{ Right hemisphere } \\
\hline & & \multirow{2}{*}{ IMEP } & \multicolumn{2}{|c|}{ cMEP } & \multirow{2}{*}{ iMEP } & \multicolumn{2}{|c|}{ cMEP } \\
\hline & & & Lat (ms) & $\operatorname{Amp}(\mu \mathrm{V})$ & & Lat (ms) & $\operatorname{Amp}(\mu \mathrm{V})$ \\
\hline \multirow[t]{4}{*}{$\mathrm{TA}$} & 13 & $\mathrm{NE}$ & $24.6 \pm 0.5$ & $460.5 \pm 151.4$ & $\mathrm{NE}$ & $25.2 \pm 0.4$ & $395.2 \pm 45.5$ \\
\hline & 14 & $\mathrm{NE}$ & $26.2 \pm 0.0$ & $346.7 \pm 40.1$ & $\mathrm{NE}$ & $27.0 \pm 0.0$ & $190.5 \pm 16.2$ \\
\hline & 15 & $\mathrm{NE}$ & $27.3 \pm 0.2$ & $253.6 \pm 31.9$ & $\mathrm{NE}$ & $26.7 \pm 0.9$ & $241.1 \pm 47.3$ \\
\hline & 19 & $\mathrm{NE}$ & $26.2 \pm 0.1$ & $132.8 \pm 41.4$ & $\mathrm{NE}$ & $26.9 \pm 0.1$ & $265.0 \pm 45.1$ \\
\hline \multirow[t]{4}{*}{ GCM } & 13 & $\mathrm{NE}$ & $25.2 \pm 0.4$ & $338.7 \pm 37.5$ & $\mathrm{NE}$ & $25.0 \pm 0.0$ & $315.2 \pm 72.7$ \\
\hline & 14 & $\mathrm{NE}$ & $25.8 \pm 0.0$ & $238.3 \pm 21.5$ & $\mathrm{NE}$ & $26.8 \pm 0.0$ & $156.3 \pm 26.6$ \\
\hline & 15 & $\mathrm{NE}$ & $26.9 \pm 0.5$ & $454.4 \pm 48.0$ & $\mathrm{NE}$ & $26.8 \pm 0.0$ & $224.0 \pm 15.5$ \\
\hline & 19 & $\mathrm{NE}$ & $26.4 \pm 0.1$ & $220.0 \pm 37.4$ & $\mathrm{NE}$ & $26.9 \pm 0.1$ & $245.0 \pm 28.9$ \\
\hline \multirow[t]{4}{*}{ VM } & 13 & $\mathrm{NE}$ & $20.4 \pm 0.5$ & $532.2 \pm 128.0$ & $\mathrm{NE}$ & $20.4 \pm 0.5$ & $431.2 \pm 41.8$ \\
\hline & 14 & $\mathrm{NE}$ & $24.0 \pm 0.0$ & $328.7 \pm 39.6$ & $\mathrm{NE}$ & $25.0 \pm 0.0$ & $278.6 \pm 30.9$ \\
\hline & 15 & $\mathrm{NE}$ & $23.5 \pm 0.5$ & $446.8 \pm 80.6$ & $\mathrm{NE}$ & $24.5 \pm 0.4$ & $422.1 \pm 21.9$ \\
\hline & 19 & $\mathrm{NE}$ & $22.9 \pm 0.1$ & $147.5 \pm 40.3$ & $\mathrm{NE}$ & $22.2 \pm 0.4$ & $122.5 \pm 12.6$ \\
\hline
\end{tabular}

Values are presented as mean \pm standard deviation.

iMEP, ipsilateral motor evoked potential; cMEP, contralateral motor evoked potential; TA, tibialis anterior; GCM, gastrocnemius; VM, vastus medialis; NE, not evoked.

\section{DISCUSSION}

To begin with, the iMEPs of distal hand muscles be- came more dominant as the patient grew older. In the arm muscles, however, it is noted that the cMEPs increased in amplitude. In those cases, the iMEP/cMEP 
A

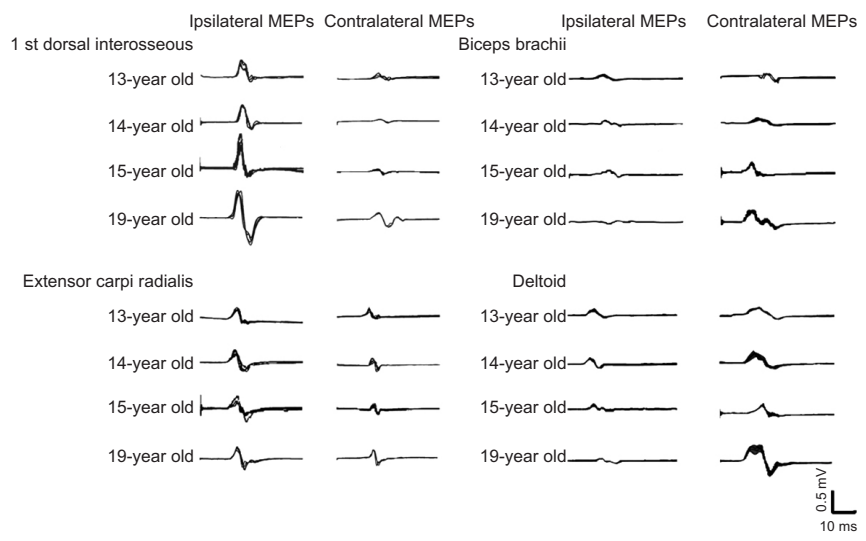

B

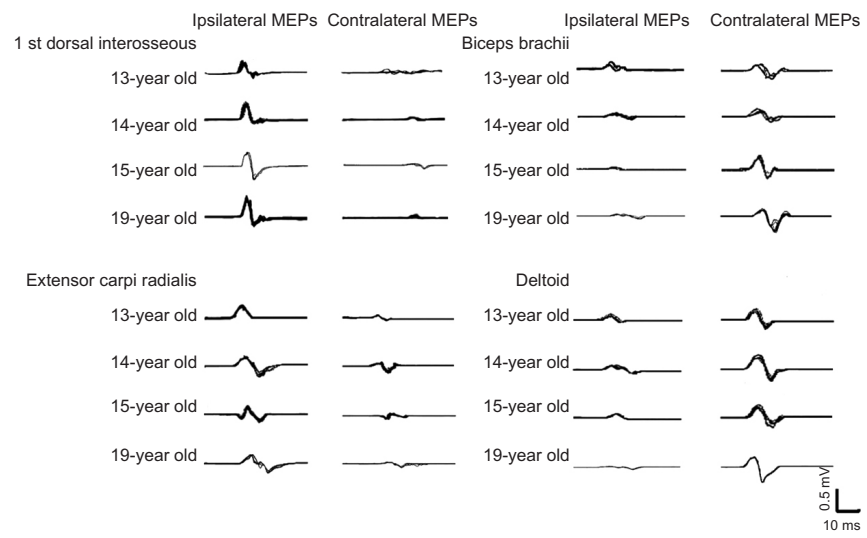

Fig. 2. Trend of motor evoked potentials (MEPs) with age, generated by transcranial magnetic stimulation at left motor cortex (A) and right motor cortex (B). For the 1st dorsal interosseous and extensor carpi radialis, ipsilateral MEPs increased in amplitude as the patient grew older. In contrast, the contralateral MEPs increased in amplitude for the biceps brachii and deltoid.

ratio was smaller in the forearm than in the distal hand, but the amplitudes of iMEPs were still larger than those of cMEPs. That being said, there was no evidence of an uncrossed corticospinal projection which was observed in the lower extremities.

In a previous TMS study of healthy children, we reported that cMEPs of distal hand muscles were noted and observed in infants. However, the cMEPs were not elicited in arm muscles, even in some children over 12 years of age. Moreover, the distal hand muscles showed a relevant amplitude increase at an earlier age than that of the arm muscles. This is a result of the late maturation of the CST projection to the proximal muscles [5]. This noted result could also be a factor in the age-related changes in the dominance of cMEPs, such as was revealed in the arm muscles that were observed in this study. Our TMS results showed that the suppression of iMEPs effects and makes cMEPs stronger, an effect that is mediated by the normal developmental pathway in the arm muscles. However, the dominance of iMEPs rather than cMEPs in the distal hand muscles represents an example of opposite changes of the motor organization pattern, as compared to the normal developmental pathway.

In our earlier report, we concluded that the iMEPs of the distal hand in our patient did not reflect the existence of a branch of a crossed CST, but rather was shown to be more characteristic of an uncrossed CST [2]. In this study we found that although the intensity of mirror movements was reduced as the patient grew older, the iMEPs of the distal hands became stronger. This finding is compatible with the previous reviewed report [1]. Moreover it suggests that an uncrossed CST represented by iMEPs are problematic for motor control, because they cannot be integrated with a crossed sensory system in that case [6].

The patient in this case had no other congenital deformity, unlike a patient we previously reported on with the characteristics of axial mesodermal dysplasia syndrome [7]. We also found that there was no genetic abnormality related to the CMMs. Although the two cases involved different causes of motor organization abnormalities, the same motor organization pattern was manifested including in this case an uncrossed corticomotoneuronal projection. This suggests that a review of the TMS studies may be more useful than only a review of genetic studies in the work confirming a diagnosis of CMM disorder.

Our TMS study challenges the validity of the Kanga mouse model of CMM disorder, which was previously used for a genetic study [8]. The Kanga mice show a unique hopping gait which reflects an uncrossed CST in the lower extremities, but our patient showed only crossed CST as characterized in the lower extremities. In addition we also question a recent DTI study which reported the existence of only an uncrossed CST in a patient with CMMs [9]. However, we confirmed not only the existence of an uncrossed CST, but also a crossed corticospinal projection to the upper extremities in our patient. Thus, further studies of more patients are definitely 
needed to reveal the underlying cause of CMMs.

\section{CONFLICT OF INTEREST}

No potential conflict of interest relevant to this article was reported.

\section{AUTHOR CONTRIBUTION}

Conceptualization: Park SH. Methodology: Kim ED, Kim GW, Won YH, Ko MH, Seo JH, Park SH. Formal analysis: Kim ED, Park SH. Funding acquisition: none. Project administration: Kim ED, Kim GW, Won YH, Ko MH, Seo JH, Park SH. Visualization: Kim ED, Park SH. Writing - original draft: Kim ED, Park SH. Writing - review and editing: Kim ED, Kim GW, Won YH, Ko MH, Seo JH, Park SH. Approval of final manuscript: all authors.

\section{REFERENCES}

1. Maegaki Y, Seki A, Suzaki I, Sugihara S, Ogawa T, Amisaki $\mathrm{T}$, et al. Congenital mirror movement: a study of functional MRI and transcranial magnetic stimulation. Dev Med Child Neurol 2002;44:838-43.

2. Park SH, Im KJ, Jo DS. Ipsilateral corticospinal projections in a patient with congenital mirror movements: a case report. J Korean Acad Rehabil Med 2009;33:5025.

3. Woods BT, Teuber HL. Mirror movements after childhood hemiparesis. Neurology 1978;28:1152-7.

4. Srour M, Riviere JB, Pham JM, Dube MP, Girard S, Morin S, et al. Mutations in DCC cause congenital mirror movements. Science 2010;328:592.

5. Yook SW, Park SH, Ko MH, Seo JH. Motor evoked potentials of the upper extremities in healthy children. Ann Rehabil Med 2011;35:759-64.

6. Son SM, Park SH, Jo DS. Ipsilateral corticospinal projections in a patient with bilateral cortical malformation: a case report. J Korean Acad Rehabil Med 2008;32:582-5.

7. Kim KW, Seo JH, Ko MH, Won YH, Park SH. A wide spectrum of axial mesodermal dysplasia complex with rhombencephalic anomaly: a case report. Ann Rehabil Med 2016;40:162-7.

8. Finger JH, Bronson RT, Harris B, Johnson K, Przyborski SA, Ackerman SL. The netrin 1 receptors Unc5h3 and Dcc are necessary at multiple choice points for the guidance of corticospinal tract axons. J Neurosci 2002;22:10346-56.

9. Brandao P, Jovem C, Brasil-Neto JP, Tomaz C, Descoteaux M, Allam N. Congenital mirror movements: lack of decussation of pyramids. Brain 2014;137(Pt 8):e292. 